\title{
Orthopädie 2000
}

Die Gesundheitsreform 2000, vorgestellt von Gesundheitsministerin Andrea Fischer, ist bei den ärztlichen Standesvertretern auf breite Ablehnung gestoßen. Letztere vertreten die Meinung, daß diese Reform gegen die Leistungserbringer, also gegen die Ärzte sei.

Wo steht unser Fach - die Orthopädie? Die demographische Entwicklung zeigt, daß immer mehr Menschen von Erkrankungen der Haltungs- und Bewegungsorgane betroffen sein werden, da wir immer älter werden. Die UNO hat das Jahr 1999 zum „Jahr des älteren Menschen“ ausgerufen. Die Lebenserwartung liegt bei uns für den heute neugeborenen Jungen bei 73 , für das neugeborene Mädchen bei 80 Jahren. Der Anteil der 60jährigen lag um die Jahrhundertwende bei $5 \%$, heute bei $21 \%$, in 8 Jahren wird er bei $26 \%$ und im Jahre 2030 bei vermutlich $38 \%$ liegen. Es steigt aber nicht nur die Lebenserwartung, sondern es steigen auch die Anforderungen an die Lebensqualität im höheren Lebensalter, Auch im „dritten“ Lebensalter möchten die Menschen mobil, sportlich aktiv ins Leben eingebunden sein.

Der medizinische Fortschritt hat es möglich gemacht, diese Wünsche und Erwartungen weitgehend zu erfüllen. durch mehr oder weniger regelmäßige Einzahlungen in eine Krankenkasse erwerben wir uns im Laufe unseres Lebens einen Anspruch auf die Leistungen, die die Medizin vorhält. Bisher haben wir in unserem System keine Einschränkung, keine Selektierung. Wie lange können wir bei der oben skizzierten Alterspyramide diese uneingeschränkte Leistungsanbietung noch durchhalten?

Und auch die jüngere Generation erwartet von der Medizin eine schnelle und vollständige Wiederherstellung der Lebensqualität. Das rupturierte Kreuzband muß nicht nur zur Wiederherstellung der Arbeitsfähigkeit repariert werden, sondern auch zur Wiedererlangung der Sportfähigkeit. Der Rückenschmerz hat unterschiedlichste Ursachen. Im sehr schnell angefertigten Computer- oder Kernspintomogramm zeigt sich eine Protrusion. Ein nach Möglichkeit minimalinvasiver Eingriff an der Bandscheibe soll alle Beschwerden heilen. Ein Kind, das mit einer körperlichen Behinderung zur Welt gekommen ist, soll durch unsere operativen Maßnahmen „normal“ werden.

Die Kosten im Gesundheitswesen sind stetig angestiegen. Durch Zunahme der Morbidität, durch die demographische Entwicklung und durch den rasanten wissenschaftlichen Fortschritt. Die Medizin wird immer leistungsfähiger. Aber bleibt sie finanzierbar? So wird die moderne Medizin zum Opfer ihres eigenen Erfolges. „In der modernen Medizin gibt es ein Bedarfsloch: Einen Übergang des theoretisch Machbaren über das praktisch Finanzierbare" (Walter Krämer). Das Machbare sollte aber auch medizinisch sinnvoll sein. Nicht alles, was uns von der Industrie als hochwirksam abgeboten wird, hält einer kritischen, wissenschaftlichen Prüfung stand. Wir Orthopäden mit unserem eher mechanistischen Denken sind auf diesem

Z. Orthop.. 137 (1999) 385

(c) 1999 Georg Thieme Verlag Stuttgart · New York
Gebiet besonders anfällig und müssen deswegen besonders auf der Hut sein. Vor allem sollten wir bei unseren $\mathrm{Pa}-$ tienten über Veröffentlichungen in der Laienpresse nicht Bedürfnisse wecken, die dann weder der einzelne noch die Gesamtheit der Ärzte erfüllen können. Im Prinzip haben wir keine Kostenexplosion im Gesundheitswesen, sondern lediglich eine Effizienz- und Leistungsexplosion. In dem Augenblick, wo eine neue Technik praktikabel wird, entsteht auch ein Bedarf.

Im Mittelpunkt aller unserer Bemühungen steht auch weiterhin der Patient. Die Zufriedenheit der Patienten erreichen wir durch eine gezielte und qualifizierte Arbeit, die sich ausrichtet an einer in der Orthopädie hohen Meßlatte. Wir müssen uns aber fragen lassen, ob alle von uns durchgeführten diagnostischen Maßnahmen immer angezeigt sind und ob wir zur Erkennung und Behandlung einer Erkrankung immer den richtigen Algorithmus wählen. Ist in der Diagnostik nicht doch das eine oder andere MRT entbehrlich?

Und wie ist es mit der Therapie? Es gibt eine Reihe von Maßnahmen, von deren Effizienz wir nicht so ganz überzeugt sind. Der wissenschaftliche Nachweis einer Wirkung fehlt. Dieses gilt besonders in der Anwendung einiger physikalischer Methoden, die erst nach ihrer breiten Einführung - wenn überhaupt - wissenschaftlich evaluiert wurden. Ähnlich ist es im operativen Bereich. Nicht jede Bandscheibenprotrusion muß operativ, auch nicht minimal-invasiv, angegangen werden. Und ob roboterunterstützte Implantationen von Hüftendoprothesenschäften eine längere Standzeit haben werden als von Hand implantierte, muß erst der Langzeitverlauf zeigen.

Wir haben in unserem orthopädischen Bereich gute Möglichkeiten, Wirksamkeit und Behandlungsnutzen zu messen. Sowohl organbezogen - durch Verbesserung der Beweglichkeit nach Implantation einer Hüfttotalendoprothese - als auch für das Individuum durch eine deutliche Verbesserung der Lebensqualität.

Die Orthopädie ist, wie oben angeführt, ein sehr weitreichendes Gebiet, das alle Lebensalter umfaßt. Die Zahl der orthopädischen Erkrankungen wird aufgrund der demographischen Entwicklung, der besseren Diagnostik und der erweiterten Behandlungsmöglichkeiten zunehmen. Wir kommen damit an eine Grenze des Finanzierbaren mit der Forderung nach evtl. Rationierung. Rationierung ist aber ein erheblicher Qualitätsverlust und würde u.U. zur viel diskutierten Zweiklassenmedizin führen. Der Patient soll die freie Wahl seines Arztes, auch seines Facharztes und seines Krankenhauses haben. Dazu bedarf es einer guten Aufklärung, auch in der Presse.

Das Fach Orthopädie wird auch im neuen Jahrtausend nicht an Bedeutung verlieren. Voraussetzung ist die Erhaltung eines hohen Standards. Dazu sind qualitätssichernde Maßnahmen erforderlich, die sich beispielsweise auch in der Erstellung der Leitlinien niederschlagen. Qualität verbessert sich durch Forschung und durch Wissen.

U. Maronna, Präsidentin der DGOT 